\title{
Laminar flow assisted anisotropic bacteria absorption for chemotaxis delivery of bacteria-attached microparticle
}

\author{
Keon $\mathrm{Huh}^{1 \dagger}{ }^{\dagger}$, Darong $\mathrm{Oh}^{1 \dagger}$, Seok Young Son ${ }^{1 \dagger}$, Hyung Jung Yoo ${ }^{1,2}$, Byeonghwa Song ${ }^{1,2}$, Dong-il Dan Cho ${ }^{1,2}$, \\ Jong-Mo Seo ${ }^{1,3}$ and Sung Jae Kim ${ }^{1,4^{*}}$
}

\begin{abstract}
The concepts of microrobots has been drawn significant attentions recently since its unprecedented applicability in nanotechnology and biomedical field. Bacteria attached microparticles presented in this work are one of pioneering microrobot technology for self-propulsion or producing kinetic energy from ambient for their motions. Microfluidic device, especially utilizing laminar flow characteristics, were employed for anisotropic attachment of Salmonella typhimurium flagellated chemotactic bacteria to $30 \mathrm{um} \times 30 \mathrm{um}$ and $50 \mathrm{um} \times 50 \mathrm{um}$ microparticles that made of biodegradable polymer. Any toxic chemicals or harmful treatments were excluded during the attachment process and it finished within $100 \mathrm{~s}$ for the anisotropic attachment. The attachments were directly confirmed by fluorescent intensity changes and SEM visualization. Chemotaxis motions were tracked using aspartate and the maximum velocity of the bacteria-attached microrobot was measured to be $\sim 5 \mathrm{um} / \mathrm{s}$ which is comparable to prior state of art technologies. This reusable and scalable method could play a key role in chemotaxis delivery of functional microparticles such as drug delivery system.
\end{abstract}

Keywords: Laminar flow, Anisotropic, Chemotaxis, Microrobot, S. typhimurium

\section{Background}

Recently, microrobots, which are capable of performing microscopic motions, have been drawn significant attentions in various fields of nanotechnology and biomedical technology due to its micro-size and controllability. Especially, due to its small size, applications of microrobots including active drug delivery system for therapeutic treatments and also a tiny lens at the tip of endoscope for diagnostic care in human body have been recently proposed [1, 2]. Microrobots, however, need suitable microactuators for propulsion and one of the major challenges are sophisticated fabrication or assembly of a proper micro and nanoscale actuator for the microrobot. In the meantime, various types of actuators have been actively

\footnotetext{
*Correspondence: gates@snu.ac.kr

${ }^{\dagger}$ Keon Huh, Darong Oh and Seok Young Son contributed equally to this work

${ }^{1}$ Department of Electrical and Computer Engineering, Seoul National University, Seoul 151-744, Republic of Korea

Full list of author information is available at the end of the article
}

investigated considering their reliability, controllability and limited size for their potential applications to these microrobots, such as an electrostatic actuator [3, 4] and a piezoelectric actuator [5], etc. While these actuators can provide a fast and controllable movement in precise manner inside human body (low Reynolds number environment in terms of fluid dynamics $[6,7])$, they either create inherent bubbles due to electrolysis, require continuous onboard power supply or are applicable only in transparent environment. Therefore, for the past few years, bioactuator based microrobot approaches have been widely studied for resolving these problems.

Bio-actuators have many advantages over artificial actuators because of self-propulsion or producing kinetic energy from ambient for their motions [8]. However, these bio-actuators inevitably have complex incubation procedures, and difficulty to integrate into objects to be manipulated using toxic chemical substances. Thus, various types of bio-actuator possessing a non-toxic substance and self-movement with no external power has

\section{Springer}

(c) 2016 Huh et al. This article is distributed under the terms of the Creative Commons Attribution 4.0 International License (http://creativecommons.org/licenses/by/4.0/), which permits unrestricted use, distribution, and reproduction in any medium, provided you give appropriate credit to the original author(s) and the source, provide a link to the Creative Commons license, and indicate if changes were made. 
extensively been studied [9]. Among these bio-actuators, the flagellated bacteria has been actively researched because the motion of the bacteria have maximum speed up to $100 \mathrm{um} / \mathrm{s}$, if required chemical resource are supplied under appropriate environment [10]. While a number of researches were performed on applying these active bacteria to bio-actuator, their toxicity and random motions prohibit further applications so that detoxifying the bacteria and boosting up the speed of a microrobot in desired direction are highly required [11-13]. For this purpose, several researchers have developed new adhesion methods for anisotropically attaching the bacteria on the microstructures considering the directionality [14], which is the main issue of the research in bacteriaattached microrobot. While one can attach the bacteria during the fabrication of the target object [15], the bacteria can be harmed due to the toxic fabrication process and it is recommended to attach the bacteria after the objects are completely assembled. Behkam et al. demonstrated $S$. marcescens flagellated chemotactic bacteria as bio-actuator for the propulsion of polystyrene microbeads by oxygen plasma treatment. With the aid of external electric field, the bacteria-attached micro-bead's motility was assessed $\left(15-30 \mathrm{um} / \mathrm{s}, E_{k}=216.522 \times 10^{-21} \mathrm{~J}\right.$ but this number contained an electrostatic energy) where $E_{k}$ is kinetic energy, which is equal to the half of mass times velocity square, that the bacteria-attached microroparticle possessed so that $E_{k}$ can consider both the motility and mass effects simultaneously [11]. Park et al. also demonstrated a conjugation method based on streptavidin/biotin reagents for strong attachment of the bacteria and microstructures by a high-affinity interaction. The bacteria-attached microparticle's motility were verified using chemotactic behavior to tumor cell $(0.5 \mathrm{um} / \mathrm{s}$, $\left.E_{k}=0.0147 \times 10^{-21} \mathrm{~J}\right)$ [16]. Sahari et al. reported anisotropic attaching the bacteria by coating poly-L-lysine to surface of the polystyrene particles with different shapes $\left(4.0 \mathrm{um} / \mathrm{s}, E_{k}=0.941 \times 10^{-21} \mathrm{~J}\right)$ [17]. Martel et al. researched a method by attaching magnetotactic bacteria to the polystyrene microbeads by immune response, producing the magnetic-guided auto-propelled microrobots. By applying electromagnetic field to the microrobot, the motility and direction was controlled $(21 \mathrm{um} / \mathrm{s}$, $E_{k}=25.936 \times 10^{-21} \mathrm{~J}$ ) [18]. However, these aforementioned methods harm the bacteria, has difficulty to modify the surface, and are often toxic for human body. Therefore, a new methodology for an effective directional control of bacteria-attached microrobot without less toxicity is required.

In this paper, we proposed a simple, but effective microfluidic structure for anisotropical absorption of bacteria to microparticles for demonstrating bacteria integrated microrobot. By flowing attenuated
Salmonella typhimurium bacteria into the microfluidic channel, hydrophobicity of microparticles made of biodegradable polycaprolacton (PCL) enabled a quick bacterial absorption $(<100 \mathrm{~s})$ without any toxic adhesive substance or chemical modification. Moreover, the bacteria were attached only at the surfaces of microcubics facing against the flow direction and the rest of the surfaces were free from the absorption, since Laminar flow can eliminate any backflow or vortical flow. The maximum motility of the bacteria-attached microparticle was measured to be $\sim 5 \mathrm{um} / \mathrm{s}\left(E_{k}=58.952 \times 10^{-21} \mathrm{~J}\right)$ by chemotaxis which is comparable to prior state of art technologies.

\section{Methods}

\section{Device fabrication}

A silicon master for poly-dimethyl siloxane (PDMS) mold was prepared as following. A 4-inch wafer was cleaned in piranha solution $\left(\mathrm{H}_{2} \mathrm{SO}_{4:} \mathrm{H}_{2} \mathrm{O}_{2}=4: 1\right)$ to remove residual organics. SU-8 2050 (MicroChem, USA) photoresist was spun (JSP4A, JD Tech, Korea). The coater parameters were set depending on the target height, for example, $500 \mathrm{rpm}$ of $15 \mathrm{~s}$ for spreading and $4000 \mathrm{rpm}$ of $40 \mathrm{~s}$ for conformal coating in case of $35 \mathrm{um}$, etc. After soft bake for $3 \mathrm{~min}$ at $95^{\circ} \mathrm{C}$, the layer was exposed by ultraviolet (UV) of $365 \mathrm{~nm}$ wavelength for $6.4 \mathrm{~s}(160 \mathrm{~mW})$ using MA-6 Aligner (Karl-suss, Germany). After post baking at $95{ }^{\circ} \mathrm{C}$ for $6 \mathrm{~min}$, the wafer was cooled down slowly to room temperature. Then the pattern was developed in PEGMA developer for $10 \mathrm{~min}$. After rinsing with isopropyl alcohol, hard bake was performed at $120{ }^{\circ} \mathrm{C}$ for 5 min. For a PDMS cast, we followed the general softlithographical fabrication processes [19]. Briefly, PDMS (Sylgard 184 Silicone elastomer kit, Dow Corning) base was mixed with a curing agent at 10:1 and bubbles were removed by vacuum jar for $1 \mathrm{~h}$. After pouring the solution onto the master, it was cured in an oven at $75{ }^{\circ} \mathrm{C}$ for $4 \mathrm{~h}$. Cured PDMS piece was peeled off from the master and cut into each device. Punching out the inlet and outlet holes was followed. Then PDMS piece and slide glass were bonded by oxygen plasma treatment (Femto Science, Korea).

\section{Microparticle fabrication}

The biodegradable PCL micro chamber was prepared using the $\mathrm{x}$-ray-lithography-based microfabrication method [20]. Briefly, a 10 \% PCL solution in di-chloromethane (DCM) was poured on a silicon wafer and coated at $1400 \mathrm{rpm}$ to obtain PCL film with the thickness of $15 \mathrm{um}$. A lamination process was used to form the double layered PCL solid film with the thickness of $30 \mathrm{um}$ [21]. X-ray synchrotron irradiation was performed at Pohang Accelerator Laboratory (PAL), Korea to pattern 
the PCL film with 30 um $\times 30$ um cubic microparticles. A $45 \%(\mathrm{w} / \mathrm{v})$ potassium hydroxide $(\mathrm{KOH})$ solution was used to separate the pattered PCL microparticles from the substrate. The microparticles were dispensed in deionized water so that the number density of the microparticles can be $10^{4}$ ea. $/ \mathrm{mL}$.

\section{Bacterial culture}

The random speed and motions of bacteria are controlled by the taxis characteristics [22]. Among various taxis mechanisms, chemotactic characteristics have been recently studied [23] because a target site (e.g. cancer site) often released a specific chemical for bacteria to be followed. S. marcescens and S. typhimurium especially shows the directional movement toward chemo-attractant by flagella motor $[24,25]$. Bacteria used in this work were attenuated S. typhimurium (SHJ2037) since it has chemotactic behavior toward a breast cancer site and also has shown a reasonable safety level by several clinical trials [26]. In addition, the attenuated S. typhimurium is characterized by fluorescence expression for easy tracking of their movements. S. typhimurium were inoculated in solid Luria-Bertani broth medium (LB medium) consisted of $500 \mathrm{~mL}$ DI water, $7.5 \mathrm{~g}$ agarose powder (DUK$\mathrm{SAN}$, Korea), $5 \mathrm{~g}$ sodium chloride $(\mathrm{NaCl}$, DUKSAN, Korea), $5 \mathrm{~g}$ tryptone (Becton, Dickinson and Company, USA) and $2.5 \mathrm{~g}$ yeast extract (Becton, Dickinson and Company, USA). The solid LB medium was incubated at $37^{\circ} \mathrm{C}$ for overnight since it is easier to get highly active bacteria in solid LB than in liquid LB. From the inoculated solid LB medium, one bacterial colony was picked and cultivated in the $10 \mathrm{~mL}$ of liquid LB medium that contained $50 \mathrm{ug} / \mathrm{mL}$ of Kanamycin and Ampicillin. Then the bacteria was incubated at $37^{\circ} \mathrm{C}$ on shacking incubator at $120 \mathrm{rpm}$ for $3 \mathrm{~h}$. The engineered S. typhimurium at an optical density $\left(\mathrm{OD}_{600}\right)$ of 1.0-1.5 (UV/VUIS Spectrophotometer-Optizen 2120 UV, Mechasys, Korea) were used in this experiment.

\section{Bacteria absorption}

Main microfluidic channel of device was connected with syringe pump (Harvard PHD 2000, USA) using four-port switching valve (IDEX, USA) for sequential injection and the outlet channel was linked with another syringe pump. First, the mixture of microparticles (100 ea./mL) was diluted with dimethyl sulfoxide (DMSO, DAEJUNG, Korea) and injected into the device at a flow rate of $0.1 \mathrm{~mL} / \mathrm{min}$ for $10 \mathrm{~min}$. DMSO reduces friction between microparticles and the surface of channel. Since DMSO has toxicity to bacteria, DI water was injected to wash out DMSO. After DMSO was completely removed, S. typhimurium was pumped into main channel at $3 \mathrm{uL} / \mathrm{s}$. The hydrophobicity of microcubics made of biodegradable
PCL enabled a bacterial absorption without any adhesive substance or chemical modification. Moreover, the bacteria can be attached only to the surfaces of microparticles facing against the flow direction and the rest of the surfaces were free from the absorption. Lastly, back-pressure from the outlet channel was applied into the main channel for releasing and collecting the bacteria-attached microparticles. All of experiments were monitored with IX51 inverted fluorescent microscope (Olympus, Japan) and the images were post processed using CellSens software (Olympus, Japan).

\section{Chemotaxis of S. typhimurium}

Aforementioned S. typhimurium was attracted by aspartate acid [27]. For evaluating the chemotactic motility, we used $10 \mathrm{mM}$ of $\alpha$-methyl-DL-aspartic acid (SigmaAldrich, USA) solution as a chemo-attractant in the flowfree microfluidic channel. The concentration of attractant was chosen as previous studies [16, 27]. Before the demonstration using the microparticles, the chemotaxis of $S$. typhimurium itself was tested in Y-shaped microchannel. The bacteria were injected from the left reservoir and $10 \mathrm{mM}$ of aspartic acid was dropped only at bottom right reservoir. See Additional file 1. While capturing this movie, bacteria were able to be moved only by diffusion since there was no net flow from anywhere. However, the bacteria tended to move toward the bottom right reservoir and there was no reason for this movement except the chemotaxis. This video clearly showed that $S$. typhimurium has the chemotaxis properties.

\section{Results and discussions}

\section{Microfluidic trapper for anisotropic bacterial absorption} Absorption concept

The main concept of the fabricating bacteria-attached microparticle is shown in Fig. 1. We designed microstructures called "trapper" to hold the microparticles for anisotropic attachment of the bacteria. The trappers play a role as structural blocker so that they allow the bacteria attachment to the surfaces of micro-cubes facing against the flow direction and, thus, the rest of the surfaces were free from the absorption. Bacteria-attached microparticle production method using the trappers is as follows. First of all, the fluid contained microparticles are injected from the top and they are caught in trappers (Fig. 1i). The shape of trapper is designed to capture only one microparticle and residual cubes automatically flow down toward the next trapper to fill the entire array of the trappers. Next, bacteria are injected for a while from the top as well. The duration and flow rate will be discussed later on. Because microparticle made of PCL polymer has hydrophobic surface [28], these surfaces adsorb bacteria without any additional chemical or physical treatments 


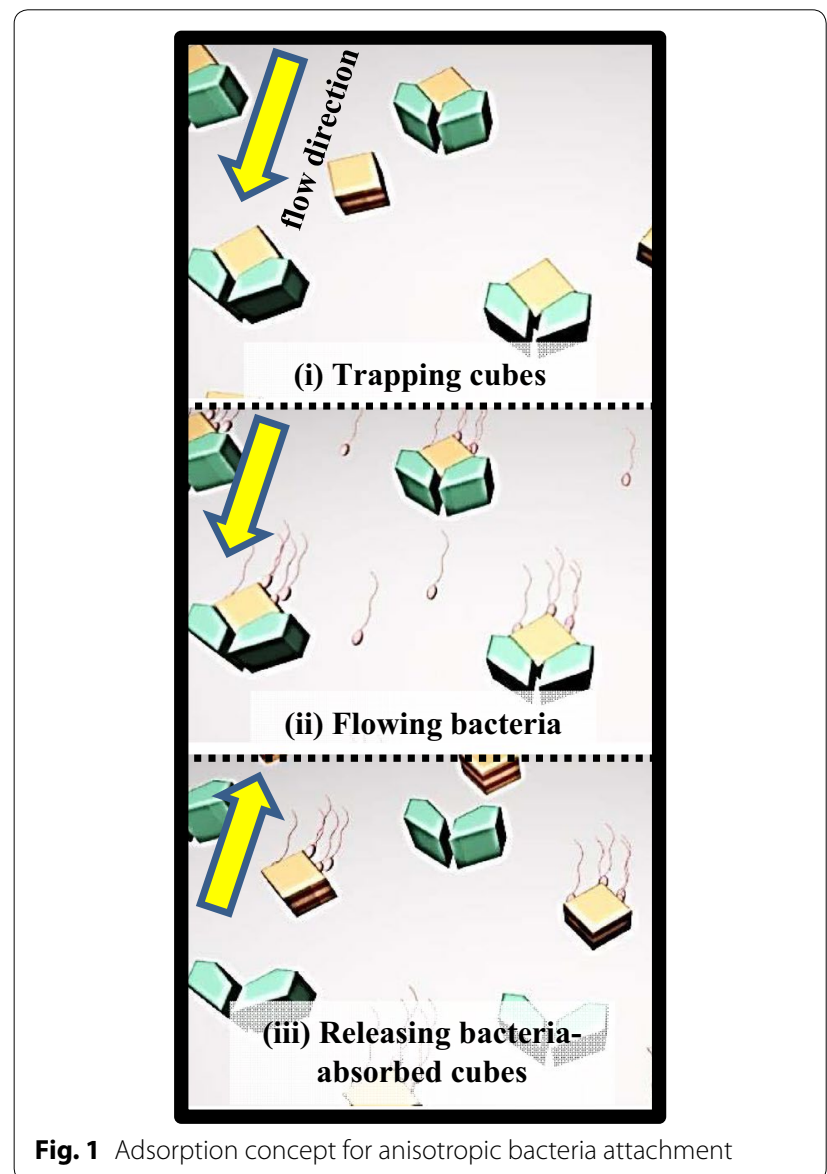

(Fig. 1ii). Excess amount of bacteria is supplied by fluidic flow to the microparticles for conformal absorption, while previous studies usually have attached the bacteria in static conditions (i.e. without any flows). The Laminar flow also helps to prevent undesirable attachment to the backside surface of microparticles. Finally, bacteria growth medium is pumped into the channel in the opposite direction (Fig. 1iii). The stream is capable of releasing the captured microparticles by fluidic inertia and they are collected at the top inlet reservoir. Thus, our unique method has several advantages; (1) simple production with low cost due to the absence of additional chemical or electrical treatments, (2) reusability of the device since severe washing steps are not involved in this concept and (3) high scalability because one can expand the size of arrays as long as low Reynolds fluidic regime is satisfied.

\section{Device design}

A simple straight channel device including trapper arrays in the middle was designed and fabricated as shown in Fig. 2a. The height of the channel was 40 or 70 um for microparticles of $30 \mathrm{um} \times 30 \mathrm{um}$ or $50 \mathrm{um} \times 50 \mathrm{um}$, respectively. The height was larger than the size of

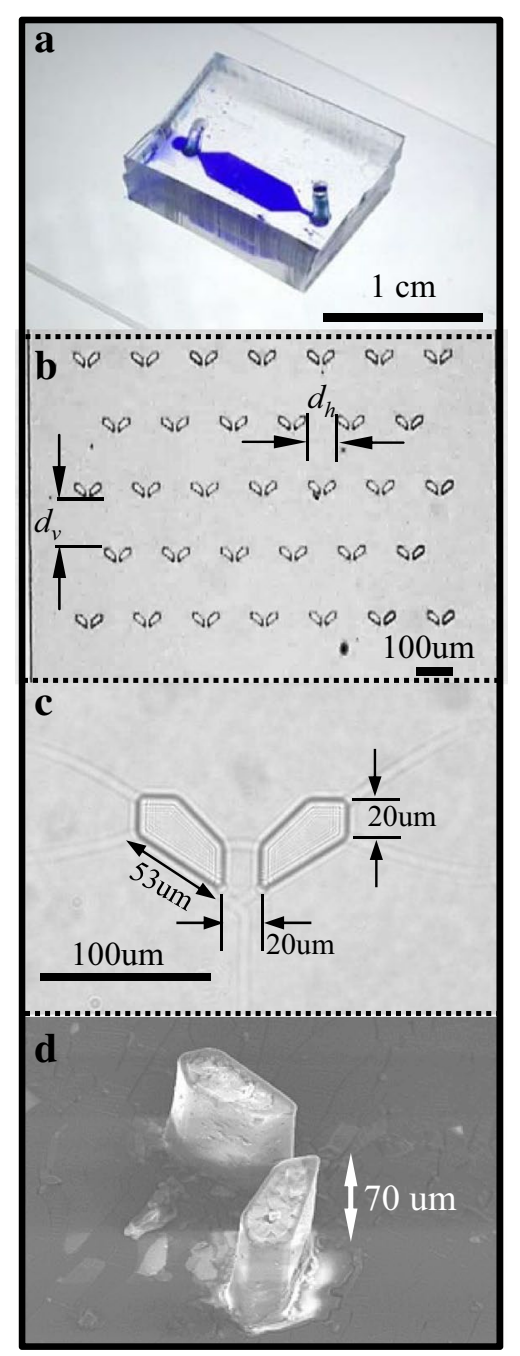

Fig. 2 a Fabricated device, $\mathbf{b}$ microscopic image of the trapper array, c magnified view for 50 um microparticle trapping and $\mathbf{d}$ SEM image of the trapper. Horizontal $\left(d_{h}\right)$, vertical $\left(d_{v}\right)$ distances and height of trapper were varied depending on the size of microparticles, while the dimension of trapper itself was fixed

microparticle for smooth flowing through the channel without clogging or stacking. The width of the channel was 2.0 or $2.5 \mathrm{~mm}$ for arranging 7-6 or 14-15 trappers in one line (Fig. 2b). The detailed shape of trapper was chevron with small gap in the middle as shown in Figs. $2 \mathrm{c}$ and 3-dimensional SEM image was shown in Fig. 2d. The gap between the trapper arms decreased fluidic resistance to assist comfort landing of microparticles. The horizontal $\left(d_{h}\right)$ and vertical $\left(d_{v}\right)$ distance between each trapper were adjusted depending the size of microparticles. $d_{h}$ was set to be 140 um (in the case of 50 um $\times 50$ um microparticle) for preventing unwanted stacking of microparticles between trappers. $d_{v}$ was determined to be $200 \mathrm{um}$ (in the case of $50 \mathrm{um} \times 50 \mathrm{um}$ microparticle) through 
analyzing data using COMSOL multiphysics. As shown in Fig. 3a, b, this microfluidic device has a low Reynold number regime so that all streamlines persist a laminar flow, i.e. the microparticles followed the streamline for consecutive trapping from the top trapper. $U_{0}$ was the inlet velocity of sample flow. However, the microparticle had significant mass so that an inertia effect drove the microparticle to fall down straightly toward the next trapper. Likewise, the microparticle went around the occupied trapper, seeking unoccupied trapper. As a result, each trapper possessed only one microparticle, filling the entire array one by one. Maximum velocity appeared at the sides of channel will be discussed later on.

Bacteria also followed the laminar streamline to approach to the microparticle. Since excess amount of bacteria were supplied from inlet reservoir, there was a higher stochastic chance for the bacteria to contact the microparticle surface than no flow condition in previous literature. However, the flow rate should be carefully determined since the bacteria should stay around the cube for the residence time (i.e. the time requires for the absorption.)

\section{Microparticle trapping efficiency}

Various dimensions of trapper were tested to capture different size of microparticles. The trapper array of $d_{h}=140$ um and $d_{v}=200$ um for $50 \mathrm{um} \times 50$ um microparticles and $d_{h}=90 \mathrm{um}$ and $d_{v}=500 \mathrm{um}$ for $30 \mathrm{um} \times$ 30 um microparticles were shown in Fig. 4a, b respectively. We considered all of one to one landing as "success" regardless of the alignment of microparticles, since anisotropic attachments still valid to the misaligned surface. The vacancy mainly occurred near both walls of microchannel. This was due to the flow rate near the walls was faster than the center of microchannel as predicted in Fig. 3. Due the fast velocity, the relative inertia of microparticles became smaller near the walls than at the center so that the microparticles tend to follow the streamline which just passes between trappers. Statistically, $70 \pm 5 \%$ of the trappers were able to capture the microparticles properly and $\sim 30 \%$ (mostly near the walls) of the trappers were unoccupied. While the success rate could be improved by adjusting a flow rate or $d_{h}$ and $d_{v}$, etc., one crucial point of this concept was one to one trapping. If more than 1 microparticle stays on the trapper, the bacteria attached only to the out-most microparticles, leading to lower the anisotropic absorption efficiency.

\section{Anisotropic bacteria absorption}

After the microparticles filled the trapper array, GFP labeled S. typhimurium were injected from the top. Figure 5a showed the snapshots during the absorption process. At first, three captured microparticles (30 um $\times$ $30 \mathrm{um})$ had almost uniform fluorescent intensity. Since the bacteria collided and were absorbed only to the two surfaces facing against the bacteria flow, the intensity of the surfaces became brighter than remaining surfaces. The intensity of the first cube started to be non-uniform from $14 \mathrm{~s}$. After $50 \mathrm{~s}$, the intensity was saturated. Therefore, the dynamics of absorption was a fast (or linear) absorption at the initial and saturation after a while (the graph of Fig. 5b). Randomly selected three different experimental sets (with the same experimental conditions) among a number of analysis were shown as a function of time and they followed the dynamics. We found that the saturation time for the anisotropic attachment was at most $100 \mathrm{~s}$ from all analyzed data sets. Compared

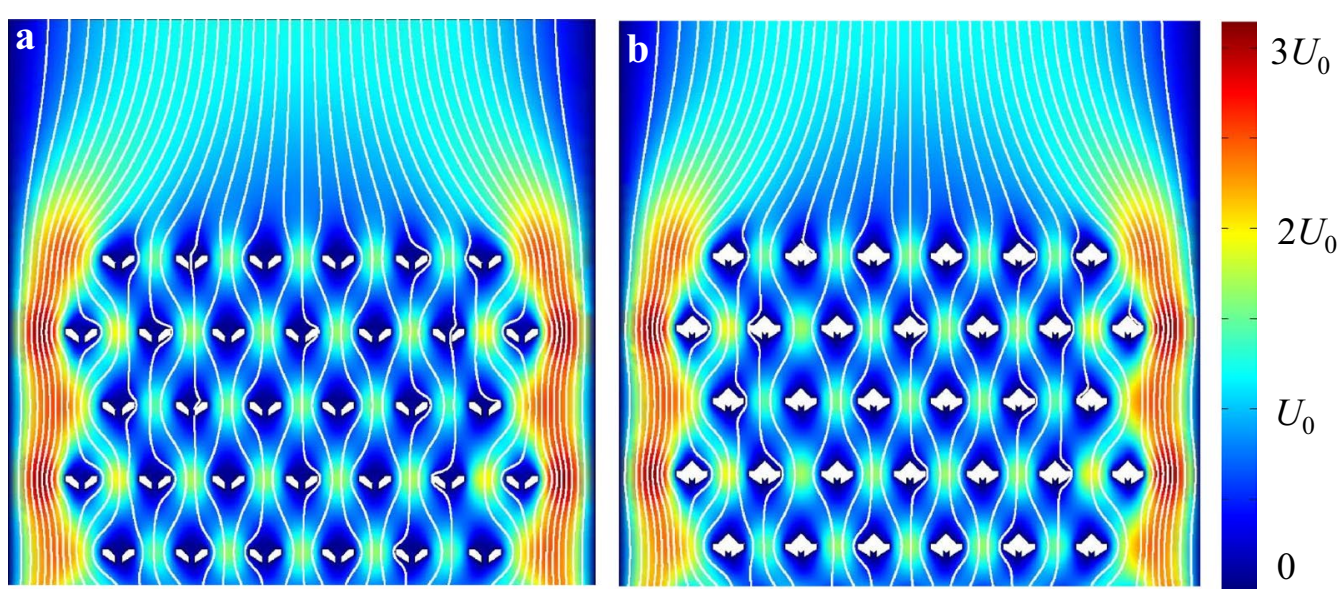

Fig. 3 Numerically simulated streamlines and velocity magnitude contour plots of (a) before and (b) after capturing microparticles. Reynolds number was set to be 1. The velocity has the maximum values near both walls of microchannel 

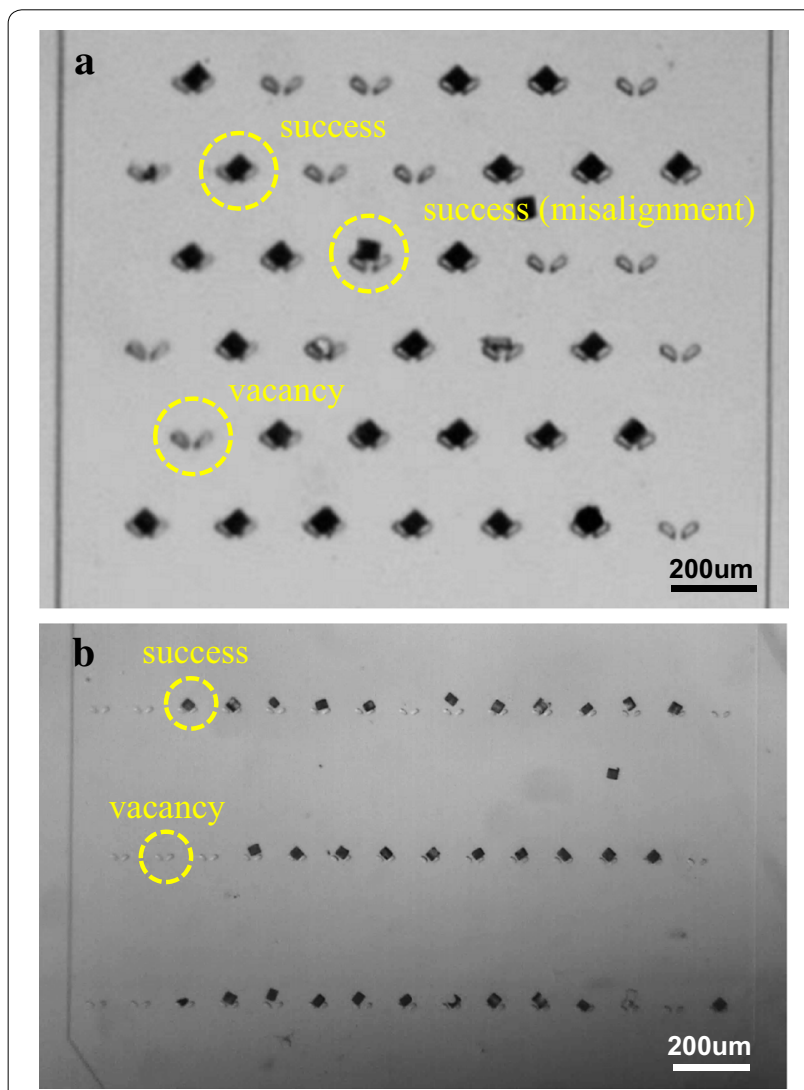

Fig. 4 Microscopic images of two different sized trappers for capturing (a) $50 \mathrm{um} \times 50 \mathrm{um}$ and (b) $30 \mathrm{um} \times 30 \mathrm{um}$ microparticles. The capturing status was categorized by success, foreign matter and vacancy. See Additional file 2

to previous studies that took at least $5 \mathrm{~min}$ for attaching bacteria, for example, S. marcescens $[29,30]$ and $V$. alginolyticus [31], even more than $30 \mathrm{~min}$ for S. typhimurium [16, 17], our laminar flow assisted method enabled faster attachment than any previous results which relied only on a random motion based (or diffusion based) attachment.

Direct evidences of the anisotropic attachment to 50 um $\times 50$ um microparticle were provided using SEM as shown in Fig. 6. Magnified view of various locations of the bacteria attached were shown as indicated i-iv. The contrast of bacteria was adjusted for clear visualization. They clearly showed that the bacteria were selectively attached at the front/left surface and the top/right surface in Fig. 6a, b, respectively. Counting the number of bacteria provided the number density of 0.3 and 0.11 ea./ $\mathrm{um}^{2}$ from each image, leading to the surface coverage of 60 and $25 \%$. The maximum number density should be limited to $0.5 \mathrm{ea} / \mathrm{um}^{2}$ since the size of bacteria is approximately $1 \mathrm{um} \times 2 \mathrm{um}$. These results were comparable to
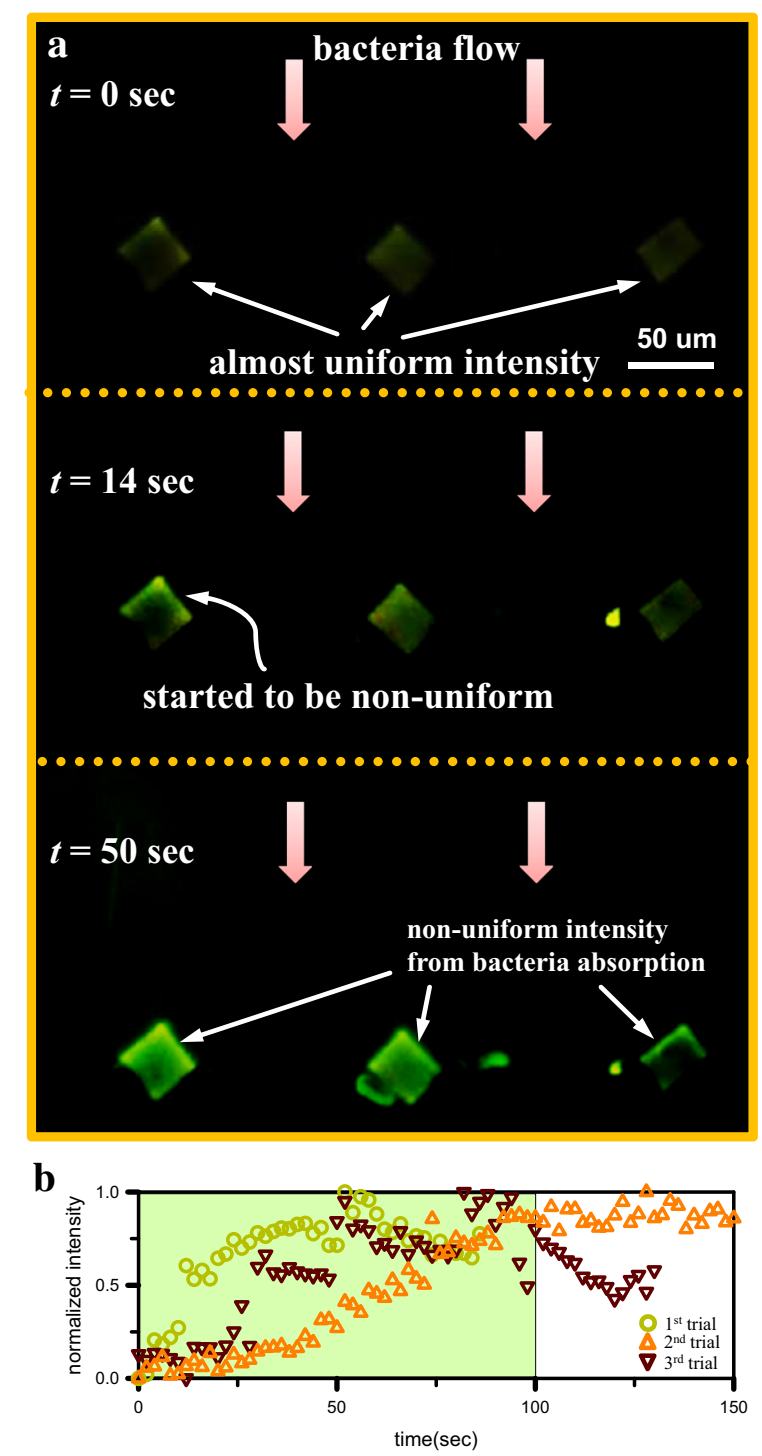

Fig. 5 a Fluorescent microscopic images of anisotropic bacteria absorption at $30 \mathrm{um} \times 30$ um microparticles. Uniform fluorescent intensity at the initial became non-uniform during the bacteria flow was being introduced from the top. $\mathbf{b}$ Saturation of fluorescent intensity at the site where the bacteria were absorbed. See Additional file 3

the state of art results, for example 0.089 and 0.1 ea./ $\mathrm{um}^{2}$ using S. marcescens [12, 32, 33], respectively. But, more efficiently, our methods achieved the results even without any toxic chemical processes during the absorption and with faster attachment time less than $100 \mathrm{~s}$ than previous studies.

\section{Chemotaxis test of bacteria-attached microparticle}

Salmonella typhimurium that was utilized in this work has chemotaxis toward the concentration 

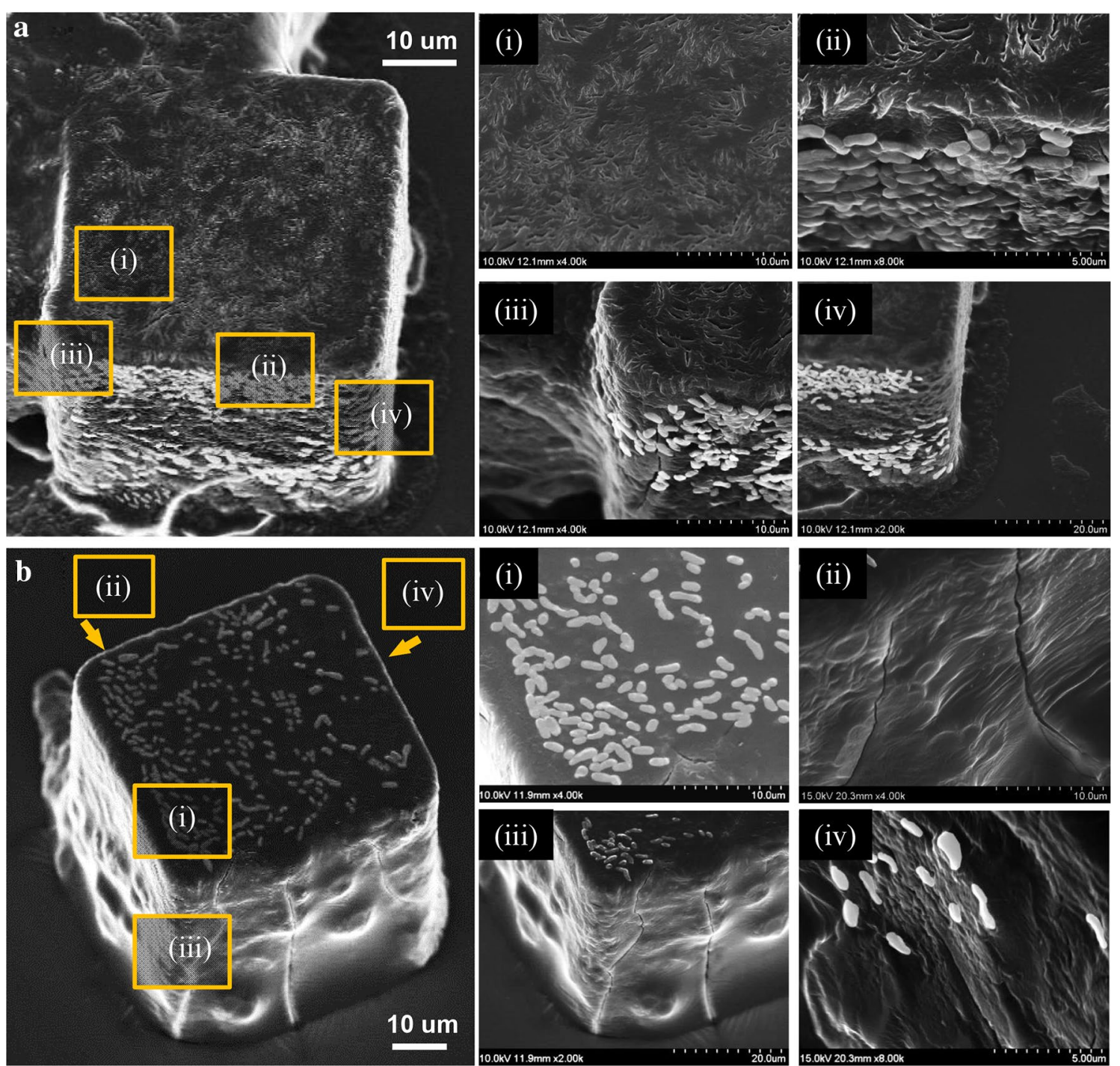

Fig. 6 SEM images of microparticles (50 um $\times 50$ um). The bacteria were selectively absorbed at (a) front/left surface and (b) top/right surface. Inset (i)-(iv) showed the magnified image of various surfaces that confirmed the anisotropic absorption

gradient of an appropriate attractant. For measuring chemotactic velocity of microparticles, we used $10 \mathrm{mM}$ aspartic acid as previous studies used [16, 27]. One representative demonstration was shown in Fig. 7 and the attractant was dropped on upper area of solution and the microparticle started to move in the direction of the concentration gradient. Images were captured every $3 \mathrm{~s}$ and it revealed that the microparticles initially moved at $10 \mathrm{um} / \mathrm{s}$ and decelerated. See Additional file 4 . The average velocity was measured to be $5 \mathrm{um} / \mathrm{s}$. The speed become zero and this is the critical evidence of no-residual flow. Slow down happens when the driving force become lower.
A constant concentration gradient is applied for the driving force in a practical chemotaxis. However, the gradient became flat due to diffusion of one-time injected aspartic acid and thus, the traveling distance in this work was only $120 \mathrm{um}$. This distance would be extended in a practical chemotaxis since there would be a constant concentration gradient. The particle was initially placed in quiescent solution inside a $5 \mathrm{~cm}$ diameter petri-dish. In such case, Reynolds number is over 100 and a residual flow, if exists, should be oscillating flow due to high inertia force. Thus, the particle must move back and forth under a residual flow, if exists. 


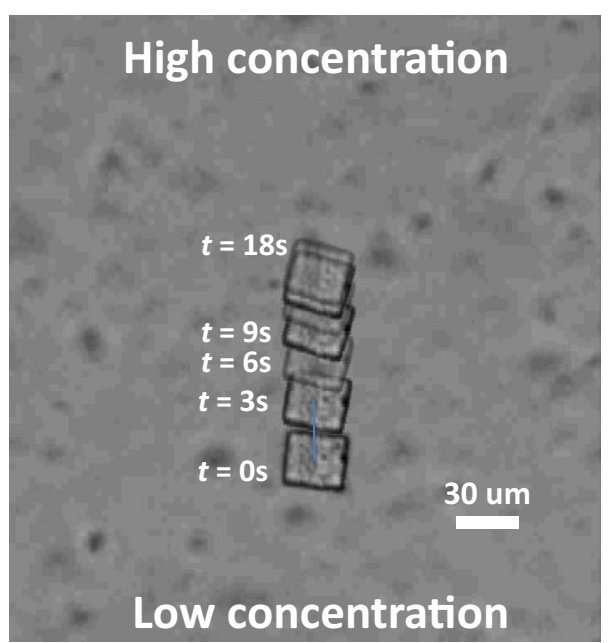

Fig. 7 Microscopic images of bacteria-attached microparticle movement. It moved in the direction of chemical concentration gradient. The images were captured every $3 \mathrm{~s}$ and overlaid. See Additional file 4

While this velocity value was considerably faster than previous bacteria-attached microparticle, one needs to have set up a proper measure, since previous studies had employed different mass (or size) of microparticles. Kinetic energy $\left(E_{k}=0.5 \mathrm{mv}^{2}\right)$ would be the candidate to investigate the efficiency of bacteria-attached microparticle. Larger mass could reduce the velocity, but could have higher $E_{k}$ value. Our presenting S. typhimurium attached microparticle has $E_{k}$ of $58.952 \times 10^{-21} \mathrm{~J}$ which is more efficient to other studies mentioned in introduction section.

\section{Conclusions}

Laminar flow assisted anisotropic attachment of S. typhimurium flagellated bacteria to the microparticles were demonstrated for a chemotactic delivery. A low cost and scalable microfluidic device was designed to trap microparticles and controlling flows upwardly/downwardly enables an anisotropic attachment of the bacteria. Direct visualizations using fluorescent intensity and SEM imaging were performed the attachment and it was measured to be less than $100 \mathrm{~s}$ to complete the absorption. Chemotaxis test revealed that the maximum velocity of the bacteria-attached microparticle was $\sim 5 \mathrm{um} / \mathrm{s}$ which is comparable to the state of art researches. Besides, this method excludes any toxic fabrication steps or chemicals so that the devices is reusable. Therefore, the presenting method is highly useful for a chemotaxis delivery of microparticles such as drug delivery system if the microparticles contains a drug. While the size of microparticle (30-50 um) are far too large to pass through the circulatory system, they are still useful when injected right near a lesion, not through a circulatory system.

\section{Additional files}

Additional file 1. Chemotaxis of S. typhimurium toward aspartic acid in Y-shape microchannel.

Additional file 2. Microscopic view of capturing $50 \mathrm{um}$ X 50um sized microparticles using trappers

Additional file 3. Fluorescent microscopic images of anisotropic bacteria absorption at $30 \mathrm{~m} \times 30$ um microparticles.

Additional file 4. Microscopic images of bacteria-attached microparticles' movement using chemical (Aspartate) concentration gradient.

\section{Authors' contributions}

$\mathrm{KH}$ and DO participated in design, fabrication and carried out testing and drafted the manuscript. SS reviewed the test results and wrote the manuscript. $\mathrm{HY}$ and BS fabricated the micro-particles that were needed on the experiment. DC and JS participated in editing the manuscript. SK guided design development, reviewed all test results, and supervised this study. All authors read and approved the final manuscript.

\section{Author details}

${ }^{1}$ Department of Electrical and Computer Engineering, Seoul National University, Seoul 151-744, Republic of Korea. ${ }^{2}$ Automation and Systems Research Institute, Seoul National University, Seoul 151-744, Republic of Korea. ${ }^{3}$ Department of Ophthalmology, Seoul National University Hospital, Seoul 110-744, Republic of Korea. ${ }^{4}$ Big Data Institute and Inter-university Semiconductor Research Center, Seoul National University, Seoul 151-744, Republic of Korea.

\section{Acknowledgements}

This work was supported by Future based Technology Development Program (Nano Fields) through the National Research Foundation of Korea (NRF) funded by the Ministry of Science, ICT and Future Planning (2012-0001033 and 2012-0009563), Basic Science Research Program (2013R1A1A1008125), the Center for Integrated Smart Sensor (CISS-2011-0031870) and Korean Health Technology RND project, Ministry of Health and Welfare, Republic of Korea (HI13C1468, HI14C0559). The author gratefully acknowledged the financial support from BK21 plus program of Creative Research Engineer Development IT, Seoul National University.

\section{Competing interests}

The authors declare that they have no competing interests.

Received: 21 October 2015 Accepted: 19 January 2016

Published online: 03 February 2016

\section{References}

1. Nelson BJ, Kaliakatsos IK, Abbott JJ (2010) Microrobots for minimally invasive medicine. Annu Rev Biomed Eng 12:55

2. Moglia A, Menciassi A, Schurr MO, Dario P (2007) Wireless capsule endoscopy: from diagnostic devices to multipurpose robotic systems. Biomed Microdevices 9:235

3. Donnelly RF, Singh TRR, Woolfson AD (2010) Microneedle-based drug delivery systems: microfabrication, drug delivery, and safety. Drug Deliv 17:187

4. Choi H, Choi J, Jang G, Park JO, Park S (2009) Two-dimensional actuation of a microrobot with a stationary two-pair coil system. Smart Mater Struct 18:055007

5. Montané E, Miribel-Catala P, Lopez-Sanchez J, Puig-Vidal M, Bota S, Samitier J (2001) High-voltage smart power integrated circuits to drive piezoceramic actuators for microrobotic applications. IEE Proc-Circuits, Devices Syst 148:343

6. Wiggins CH, Goldstein RE (1998) Flexive and propulsive dynamics of elastica at low Reynolds number. Phys Rev Lett 80:3879 
7. Becker LE, Koehler SA, Stone HA (2003) On self-propulsion of micromachines at low Reynolds number: purcell's three-link swimmer. J Fluid Mech 490:15

8. Darnton N, Turner L, Breuer K, Berg HC (2004) Moving fluid with bacterial carpets. Biophys J 86:1863

9. Akiyama Y, Odaira K, Sakiyama K, Hoshino T, Iwabuchi K, Morishima K (2012) Rapidly-moving insect muscle-powered microrobot and its chemical acceleration. Biomed Microdevices 14:979

10. Sowa Y, Berry RM (2008) Bacterial flagellar motor. Q Rev Biophys 41:103

11. Behkam B, Sitti M (2008) Effect of quantity and configuration of attached bacteria on bacterial propulsion of microbeads. Appl Phys Lett 93:223901

12. Park SJ, Bae H, Kim J, Lim B, Park J, Park S (2010) Motility enhancement of bacteria actuated microstructures using selective bacteria adhesion. Lab Chip 10:1706

13. Yoo H, Lee S, Cho D-I (2014) Motility control of bacteria-actuated biodegradable polymeric microstructures by selective adhesion methods. Micromachines 5:1287

14. Park SJ, Bae H, Ko SY, Min J-J, Park J-O, Park S (2013) Selective bacterial patterning using the submerged properties of microbeads on agarose gel. Biomed Microdevices 15:793

15. Steager EB, Sakar MS, Kim DH, Kumar V, Pappas GJ, Kim MJ (2011) Electrokinetic and optical control of bacterial microrobots. J Micromech Microeng 21:035001

16. Park SJ, Park SH, Cho S, Kim DM, Lee Y, Ko SY, Hong Y, Choy HE, Min JJ, Park JO, Park S (2013) New paradigm for tumor theranostic methodology using bacteria-based microrobot. Sci Rep 3:3394

17. Sahari A, Headen D, Behkam B (2012) Effect of body shape on the motile behavior of bacteria-powered swimming microrobots (BacteriaBots). Biomed Microdevices 14:999

18. Martel S, Tremblay CC, Ngakeng S, Langlois G (2006) Controlled manipulation and actuation of micro-objects with magnetotactic bacteria. App Phys Lett 89:233904

19. Xia Y, Whitesides GM (1998) Soft lithography. Annu Rev Mater Sci 28:153
20. Dong-il Dan C, Hyung Jung Y (2015) Microfabrication methods for biodegradable polymeric carriers for drug delivery system applications: a review. Microelectromech Syst 24(1):10-18

21. Yoo H, Hong S, Lee S, Ahn J, Cho D (2012) Fabrication of laminated biodegradable polymeric structure for controlled drug release. Proc. of KMEMS FP-2-39

22. Blair DF (1995) How bacteria sense and swim. Annu Rev Microbiol 49:489

23. Wadhams GH, Armitage JP (2004) Making sense of it all: bacterial chemotaxis. Nat Rev Mol Cell Biol 5:1024

24. Zhulin IB, Rowsell EH, Johnson MS, Taylor BL (1997) Glycerol elicits energy taxis of Escherichia coli and Salmonella typhimurium. J Bacteriol 179:3196

25. Zhuang J, Wei G, Carlsen RW, Edwards MR, Marculescu R, Bogdan P, Sitti M (2014) Analytical modeling and experimental characterization of chemotaxis in Serratia marcescens. Phys Rev E 89:052704

26. Min JJ, Nguyen VH, Kim HJ, Hong Y, Choy HE (2008) Quantitative bioluminescence imaging of tumor-targeting bacteria in living animals. Nat Protoc 3:629

27. Choi E, Chang HK, Young Lim C, Kim T, Park J (2012) Concentration gradient generation of multiple chemicals using spatially controlled selfassembly of particles in microchannels. Lab Chip 12:3968

28. Eisenbach M (1996) Control of bacterial chemotaxis. Mol Microbiol 20:903

29. Behkam B, Sitti M (2007) Bacterial flagella-based propulsion and on/off motion control of microscale objects. Appl Phys Lett 90(2):023902

30. Kim D, Liu A, Diller E, Sitti M (2012) Chemotactic steering of bacteria propelled microbeads. Biomed Microdevices 14:1009

31. Nogawa K, Kojima M, Nakajima M, Homma M, Arai F, Fukuda T (2011) Smart manipulation of multiple bacteria-driven microobjects based on bacterial autonomous movement. IEEE Intelligent Robots and System

32. Behkam B, Sitti M (2009) Characterization of bacterial actuation of microobjects (IEEE Intelligent Robots and System)

33. Edward BS, Mahmut Selman S, Dal Hyung K, Vijay K, George JP, Min Jun K (2011) Electrokinetic and optical control of bacterial microrobots. J Micromech Microeng 21:035001

\section{Submit your manuscript to a SpringerOpen ${ }^{\odot}$ journal and benefit from:}

- Convenient online submission

- Rigorous peer review

- Immediate publication on acceptance

- Open access: articles freely available online

- High visibility within the field

- Retaining the copyright to your article

Submit your next manuscript at $>$ springeropen.com 